\title{
3D imaging of the elemental concentration associated with a malignant tumor in breast cancer using Neutron Stimulated Emission Computed Tomography: a Monte Carlo simulation study
}

\author{
N. Araghian, H. Miri-Hakimabad ${ }^{\star}$ and L. Rafat-Motavalli \\ Physics Department, College of Sciences, Ferdowsi University of Mashhad, 91775-1436, Mashhad, Islamic Republic of Iran.
}

Received 8 July 2015 - Accepted 4 March 2016

\begin{abstract}
Three-dimensional imaging of a cancerous breast with NSECT was proposed for the first time in this study. An irradiation system in a clinically relevant situation was simulated using the MCNP code. In the reconstructed images, the location and boundary of the tumor were identified by four elements, especially by ${ }^{39} \mathrm{~K}$, with the ratio of malignant to healthy pixel intensity of about 2.446 ( $p$-value $\ll 0.01$ ). The average glandular dose was lower than the reference levels, and was comparable with the dose of digital X-ray mammography and 3D tomosynthesis examinations.
\end{abstract}

Keywords: neutron stimulated emission computed tomography / breast cancer imaging / early diagnosis / Monte Carlo simulation / MLEM reconstruction

\section{Introduction}

Breast cancer is one of the major causes of cancer-related morbidity and mortality among women worldwide (Pedraza et al., 2010). In Middle East countries, breast cancer is the most frequent cancer in women, comprising $21.4 \%$ of all malignancies in females (Harirchi et al., 2004). The main problems for women in these countries compared with women in developed countries are late diagnosis and occurrence at a younger age (at least one decade later than 50). In the area of breast cancer diagnosis, the common imaging modality to monitor the breast disease and improve the chance of treatment for asymptotic women is screening mammography (Leitch et al., 1997). Digital breast tomosynthesis as an alternative to the traditional 2D mammography is suggested for screening breast cancer. However, the use of this technique in a clinical setting is still under investigation (Baptista et al., 2014). Up to now, mammography has had many successes in reducing breast cancer mortality. Nevertheless, it has been associated with some limitations (Van Schoor et al., 2011).

Indeed, the resolution of mammogram images is dependent on breast density. A radiographically dense breast, usually found in women younger than 40 years, increases the risk of cancer and makes mammograms less accurate (Boyd et al., 2005, 2007; McCormack and dos Santos, 2006). Thus, mammography is insufficient to screen large groups of young people. Moreover, to distinguish the tumor from the healthy tissue in mammography, it must be significantly more dense than the healthy tissue. Hence, in addition to mammography, a biopsy

\footnotetext{
* mirihakim@ferdowsi.um.ac.ir
}

is often required to investigate the malignancy of a suspected lesion.

Due to these limitations, the study of other biomarkers such as trace elements existing in cancerous tissue has been suggested for early diagnosis through the Neutron Stimulated Emission Computed Tomography (NSECT) method (Floyd Jr et al., 2006; Bender et al., 2007; Kapadia, 2011; Kapadia et al., 2008, 2010; Viana et al., 2013). The main idea of this method is based on detecting fluctuations in the value of trace elements between normal and malignant breast tissue at different stages of tumor development. These fluctuations have been observed in several studies for breast cancer (Schwartz, 1975; Ng et al., 1997; Siddiqui et al., 2006; Farquharson et al. 2007) and also in other diseases (Lovell et al., 1998; Yaman et al. 2005).

The primary investigations of breast cancer diagnosis with NSECT were based on spectroscopic studies. The feasibility of spectroscopic detection of breast cancer markers was studied by Bender et al. (2007) in a simple geometric sample. To evaluate the sensitivity of the NSECT method, Kapadia et al. (2008) experimentally tried to detect the elements which could be identified through Neutron Activation Analysis at a microgram level of concentration. After that, the ability to generate two-dimensional images of breast tumors was examined by Kapadia et al. (2010) using some elements' signatures. It was also found that neutron beam spectroscopy based on NSECT can be used to evaluate the change in the calcium isotopic composition due to the development of breast microcalcifications (Viana and Horiyaz, 2011).

Following these efforts to make NSECT an in vivo clinical breast screening technique, in this article, the feasibility of 


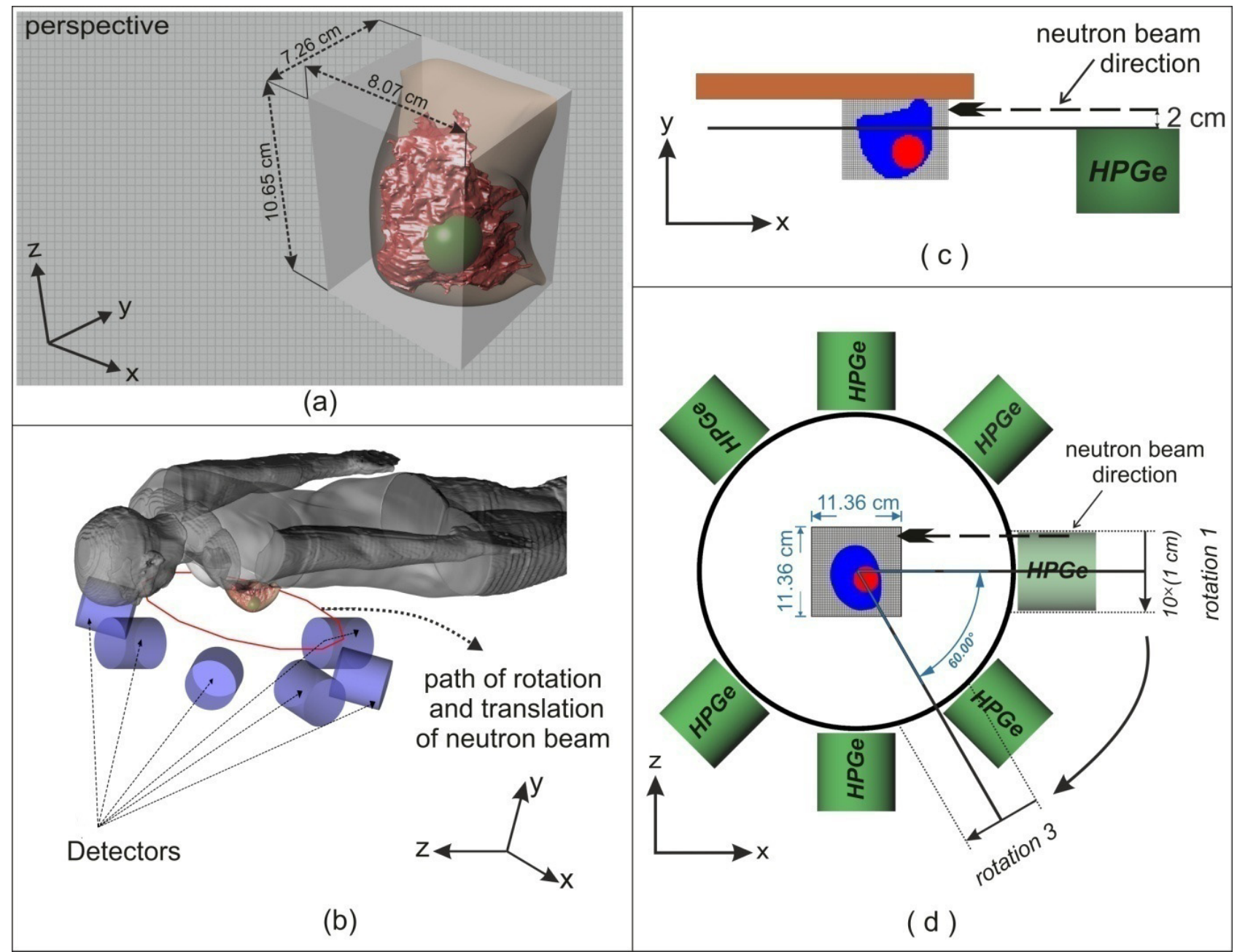

Figure 1. The schematic view of the breast phantom and simulated imaging system: (a) breast phantom. Dimensions of the box surrounded the breast are $8.1 \mathrm{~cm} \times 7.3 \mathrm{~cm} \times 10.7 \mathrm{~cm}$ (width $(\mathrm{x}) \times$ depth $(\mathrm{y}) \times$ height $(\mathrm{z})$ ). (b) Perspective view of the simulated imaging system. (c) Top view of imaging system. The set of detectors was always positioned in the half-space in front of body. Each detector had a $5 \mathrm{~cm}$-diameter cylinder with $10 \mathrm{~cm}$ height and was entirely filled with natural germanium of $5.32 \mathrm{~g} \mathrm{~cm}^{-3}$. The plane of them is $2 \mathrm{~cm}$ below the neutron beam plane. (d) The frontal view of imaging system. The plane of irradiation is observed in this view. The neutron beam was rotated around the circle with radius of $20 \mathrm{~cm}$. The first step is noted as "rotation 1", which is turned to "rotation 2" and after "rotation 3" by two successive 30-degree shifts and so on. Dashed arrow line shows the direction of beam. At each rotation step, the beam was transferred in 10 translated steps. The direction of translating is depicted by solid arrow line. The detectors were positioned in $0^{\circ}, \pm 45^{\circ}, \pm 90^{\circ}$ and $\pm 135^{\circ}$ with respect to the beam direction.

3D imaging of trace elements concentrated in the breast was proposed in order to identify the malignancy and determine spatial information such as the size, true boundary and location of tumors. Monte Carlo simulations were used to model a realistic breast sample imaging with NSECT, which provided a $2 \mathrm{D}$ view of the elemental concentration of the organ at different projections. Then, starting from the profiles of different sections, a $3 \mathrm{D}$ view of the breast was created.

\section{Materials and methods}

\subsection{Simulation of the phantom}

The adult female phantom, simulated in a prone posture with a pendant breast (Araghian et al., 2015), was used for imaging and dosimetry calculation. The breast of the phantom contained a malignant tumor. According to TNM breast cancer staging, invasive breast cancer starts from stage II, in which the size of the tumor is larger than $2 \mathrm{~cm}$ but not larger than $5 \mathrm{~cm}$. Therefore, for the purpose of this study, the tumor was simulated with a diameter of $3 \mathrm{~cm}$, which is large enough to exhibit developing a lesion to the malignancy stages of breast cancer. The breast sample is shown in Figure 1a.

The percentage of breast glandularity of the phantom was about $40 \%$. However, the glandular and adipose tissues of the breast were uniformly filled with healthy tissue composition. The healthy and malignant breast tissue were simulated with a density of 0.95 (ICRP Publication 89, 2002) and $1.058 \mathrm{~g} \mathrm{~cm}^{-3}$, respectively (Bender et al., 2007), and their elemental composition is shown in Table 1 (Ng et al., 1997; Bender et al., 2007). 
Table 1. Material composition from ( $\mathrm{Ng}$ et al., 1997) for healthy and malignant tissues. The breast phantom and tumor were uniformly filled according to the second and third column, respectively.

\begin{tabular}{lccc}
\hline & Healthy (\%) & Malignant (\%) & Malignant /Healthy (per unit volume of breast) \\
\hline Oxygen & $6.14 \mathrm{E}+01$ & $6.14 \mathrm{E}+01$ & 1.114 \\
Carbon & $2.29 \mathrm{E}+01$ & $2.29 \mathrm{E}+01$ & 1.114 \\
Hydrogen & $1.26 \mathrm{E}+01$ & $1.25 \mathrm{E}+01$ & 1.102 \\
Nitrogen & $2.57 \mathrm{E}+00$ & $2.57 \mathrm{E}+00$ & 1.114 \\
Chlorine & $1.98 \mathrm{E}-01$ & $2.15 \mathrm{E}-01$ & 1.210 \\
Sodium & $1.85 \mathrm{E}-01$ & $2.00 \mathrm{E}-01$ & 1.204 \\
Potassium & $8.94 \mathrm{E}-02$ & $1.96 \mathrm{E}-01$ & 2.442 \\
Iron & $9.80 \mathrm{E}-03$ & $7.85 \mathrm{E}-03$ & 0.892 \\
Calcium & $8.29 \mathrm{E}-03$ & $1.13 \mathrm{E}-02$ & 1.518 \\
Zinc & $1.17 \mathrm{E}-03$ & $1.14 \mathrm{E}-03$ & 1.085 \\
Bromine & $7.07 \mathrm{E}-04$ & $6.55 \mathrm{E}-04$ & 1.032 \\
Aluminum & $6.67 \mathrm{E}-04$ & $5.70 \mathrm{E}-04$ & 0.952 \\
Rubidium & $5.98 \mathrm{E}-04$ & $5.84 \mathrm{E}-04$ & 1.088 \\
Manganese & $3.88 \mathrm{E}-05$ & $3.16 \mathrm{E}-05$ & 0.907 \\
Cobalt & $2.06 \mathrm{E}-05$ & $1.98 \mathrm{E}-05$ & 1.071 \\
Cesium & $3.27 \mathrm{E}-07$ & $3.60 \mathrm{E}-07$ & 1.226 \\
\hline
\end{tabular}

\subsection{Simulation of the imaging system}

An NSECT imaging system includes a neutron beam and gamma-ray detectors. The neutron beam energy was set to $5 \mathrm{MeV}$ to excite most of the trace elements in the sample. The gamma-ray detectors were simulated as high-purity germanium (HPGe) detectors with an effective resolution of $0.1 \%$ at $1 \mathrm{MeV}$ associated with little change in the energy range of 0.5 to $6.5 \mathrm{MeV}$, which are used in NSECT (Knoll, 2010).

They were arranged around the breast, reproducing a realistic imaging situation with the patient in the prone posture. Therefore, only half the space beyond the chest wall or in front of the breast was available to position the detectors. As shown in Figure 1c, a set of gamma-ray detectors was positioned on a plane $2 \mathrm{~cm}$ below the beam plane. Following the findings of previous work on NSECT, the polar positions of the detectors with respect to the beam direction were set at $\pm 45^{\circ}$ and $\pm 135^{\circ}$, where the maximum emission takes place for several elements of breast materials (Kapadia, 2011; Kapadia et al., 2008, 2010).

In order to increase tomographic samplings or gamma-ray events per projection, two additional HPGe detectors were positioned at $0^{\circ}$ and $\pm 90^{\circ}$, while the angle of $180^{\circ}$ (i.e. in front of the beam direction) was excluded to prevent damaging the crystal of the detector subject to neutron interactions.

\subsection{Tomographic acquisition}

The present simulated geometry was similar to firstgeneration CT scanners. The imaging system was rotated around the breast sample, in 6 angles over $180^{\circ}$ with respect to the neutron beam direction. Within each angle of rotation, the 1-cm-width beam was translated in 10 steps so that the area proportional to the length of the FOV edge $(11.36 \mathrm{~cm})$ was illuminated while the beam aperture had no overlapping in consecutive displacements. Consequently, 60 beam projections were created per slice. At each projection, the line of collimated neutrons (LOC) illuminated a part of the FOV and then, gamma-ray detectors recorded the data related to this line. The irradiation plane was parallel to the coronal plane of the body (x-z plane in Figure 1b), i.e. the beamlines and torso did not collide.

After completing the scan process in one slice (x-z), the total imaging system was translated to the next tomographic slice along the y-axis. Similarly, the number of tomographic slices was adjusted to the beam width through which the volume of breast sample could be completely scanned. Consequently, for the size of our sample, there were 7 slices in the y direction. Figures $1 \mathrm{c}$ and $1 \mathrm{~d}$ show the scheme of the beam and detectors around the breast.

\subsection{Monte Carlo calculations}

Monte Carlo simulations were made using MCNPX 2.6 code. The ENDF/B-VII libraries were used for tracking neutrons and their secondary gamma rays (Chadwick et al., 2006). The voxel format of the phantom was incorporated into a MCNP lattice file. The dimensions of each voxel were $0.1775 \mathrm{~cm} \times 0.1775 \mathrm{~cm} \times 0.484 \mathrm{~cm}$ (width $(\mathrm{x}) \times$ depth $(\mathrm{y}) \times$ height (z)).

The mesh tally type 1 with the 'flux' keyword, f1:p and f6:n,p tally were used to calculate two independent terms of the response matrix of the image reconstruction process (as will be defined in Section 2.5.1), the gamma-ray spectrum of the breast sample recorded by the detectors at each projection, and the breast and total-body doses, respectively.

To calculate the gamma-ray spectrum of the breast sample, the energy range of the gamma-ray events, registered over the detector's surfaces, was 1 to $10 \mathrm{MeV}$. The energies were binned in $1 \mathrm{keV}$, which was adjusted to the resolution of the HPGe detector (Knoll, 2010).

The total number of MCNP running programs was about 88,000 , which required an execution time of about 6700 hours 
per processing core. In these simulations, the number of histories was large enough to maintain a statistical uncertainty within $5 \%$ for all calculations.

\subsection{Image analysis}

This section describes the process of extracting the images required to identify the tumor.

\subsubsection{Image reconstruction}

For each rotation-translation step of the imaging system, gamma spectra were recorded by the detectors. Then, the pulse heights related to the characteristic gamma rays of each element were extracted to form a sinogram, which was used to reconstruct the image of elemental concentration using a maximum-likelihood expectation-maximization (MLEM) algorithm in an array of $64 \times 64$ pixels (Dempster et al., 1977; Agasthya et al., 2011).

In order to estimate the unknown pixel intensities in the process of image reconstruction, an operating equation is solved through different algorithms such as MLEM. In this equation, a projection operator called the response matrix relates the characteristic events recorded by gamma-ray detectors at different projections (projection values) to the number of gamma rays emitted from each pixel of the FOV (intensity of pixels).

In NSECT, the response matrix is interpreted as the probability of producing a gamma photon through the interaction of neutrons with the isotope inside the voxel $j$ and recording the resulting gamma rays in projection $i$, where $j$ and $i$ are numbered based on the total voxels at the FOV $\left(n_{p i x}\right)$ and total projections $\left(n_{p r o j}\right)$, respectively. Therefore, each array of the response matrix is the product of two terms. The first term is the probability of neutron interactions with the elements in a specific pixel of the FOV at a specific projection. This is proportional to the track length estimation (or neutron flux) of every neutron entering that pixel. The second term is the probability of recording the gamma rays produced in a given pixel by the detectors, which is proportional to the amount of gamma rays incoming to the detectors' surfaces.

According to this definition, each response matrix only belongs to the characteristic gamma rays corresponding to a specific element. Hence, before calculating the response matrix, the appropriate elements must be selected to reconstruct the images.

\subsubsection{Candidate elements for tumor identification}

In this study, the trace elements were accepted if they could satisfy the following conditions: 1) among all trace elements, they were present in higher concentrations in both normal and malignant tissues, and 2) the pulses of their characteristic gamma rays were distinguishable in the gamma spectra of both normal and malignant tissue. For this purpose, two simple spherical samples containing the healthy and malignant elemental compositions were irradiated by $5 \mathrm{MeV}$ neutrons. Then, the resulting gamma-ray spectra were analyzed in an ideal $4 \pi$ HPGe detector.

\subsubsection{Volume rendering}

In order to create a three-dimensional image, to separate the tumor from the breast and to calculate the volume of each region, the set of two-dimensional reconstructed images must be segmented. For segmenting with high accuracy, the visual representation of different regions of interest (ROIs) in the images was enhanced by applying a binary mask to all images after the pixel intensities were converted to grayscale. The pixel intensities of images (or gray values) are proportional to the concentration of elements in different regions of the FOV. By applying the binary mask, pixel intensities give logical values in such a way that pixel intensities above the mask threshold are white. The appropriate threshold was determined through an iterative process so that the total volume of white pixels was adjusted to the volume of the region of interest - i.e. breast or tumor - with high accuracy (more than 99.99\%). In this way, discriminating the absolute light from absolute dark pixels was simpler for the segmentation of different regions.

After determining the threshold, the binary image representing the breast was combined with the binary image of the tumor to create a combined image containing both the breast and the tumor.

Image segmentation and volume rendering was done using 3D-DOCTOR ${ }^{\mathrm{TM}}$ (Able Software Corp., Lexington, MA). Multiple slices of images in DICOM format were imported into 3D-DOCTOR to create a 3D image stack. The boundaries of the tumor and breast were contoured and smoothed. Then, the polygon-mesh model was extracted using the complex surface rendering function to create the 3D image of the cancerous breast. Finally, the location and volume of the tumor in the $3 \mathrm{D}$ model were calculated and compared against that in the voxel-based breast phantom.

\subsection{Metric parameters for determining the malignancy}

To determine the malignancy and detectability of a lesion within the reconstructed images, the concentration levels of trace elements were calculated quantitatively using the contrast, contrast to noise ratio (CNR) and the ratio of mean pixel intensity between the tumor and breast within the same chosen ROI.

In addition, the location and boundary of the tumor in the reconstructed images were compared with the irradiated layouts of the voxel-based breast phantom that were assumed to be reference images. Since different regions of the breast phantom had different concentrations of trace elements, reference images were assumed to have three nominal values of pixel intensity, being proportional to the elemental concentration in the tumor, breast and outer region of the breast. For instance, the nominal values of pixel intensity in reference images related to the potassium concentration were considered to be 1 (for the tumor region), 0.41 (for the breast region) and 0 (for outside the breast region). Therefore, the changes in pixel intensity in these images would show a vertical step trend. 


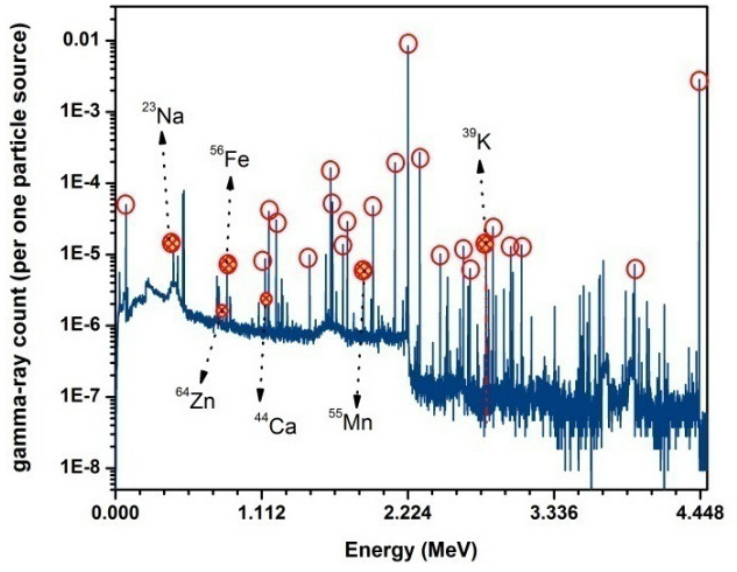

(a)

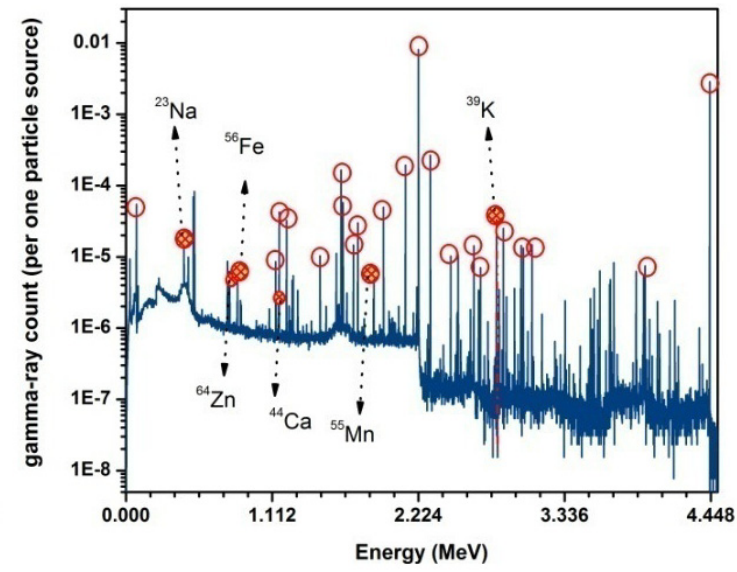

(b)

Figure 2. Gamma energy spectrum from (a) normal and (b) malignant breast spherical samples showing energy peaks for several potential elements identified. The peaks marked by solid circles belong to the isotopes of ${ }^{23} \mathrm{Na},{ }^{64} \mathrm{Zn},{ }^{56} \mathrm{Fe},{ }^{44} \mathrm{Ca},{ }^{55} \mathrm{Mn}$ and ${ }^{39} \mathrm{~K}$, which can be detected. The other peaks marked by hollow circles were generated from the neutron interaction with chlorine, nitrogen, hydrogen and carbon. They produce a broad background throughout the spectrum. The peaks of $2.223 \mathrm{MeV}$ (neutron capture by ${ }^{2} \mathrm{H}$ ) and $4.438 \mathrm{MeV}$ (inelastic scattering of neutron by ${ }^{12} \mathrm{C}$ ) have the maximum values. The different concentration levels of ${ }^{39} \mathrm{~K}$ between malignant and normal tissues in energy of $2.814 \mathrm{MeV}$ can be clearly observed.

\section{Results}

\subsection{Candidate elements}

Figure 2 shows the gamma-ray spectra of normal and malignant spherical samples. The energy of gamma rays was matched to the corresponding elements using both the gamma energy listed on NUDAT ${ }^{1}$ and the MCNPX simulation. Most of the peaks were generated from the regular elemental composition of the breast, such as hydrogen, nitrogen and carbon. Meanwhile, the characteristic gamma rays due to neutron capture interaction with chlorine such as 1.131, 1.165, $1.648,1.959$ and $6.111 \mathrm{MeV}$ were observed in the gamma-ray spectra of the samples. These peaks spread a high background throughout the spectrum.

The peaks marked by solid circles are observable for trace elements. They belong to the isotopes of ${ }^{23} \mathrm{Na},{ }^{64} \mathrm{Zn}$, ${ }^{56} \mathrm{Fe},{ }^{44} \mathrm{Ca},{ }^{55} \mathrm{Mn}$ and ${ }^{39} \mathrm{~K}$. By looking at Table 1, it can be seen that sodium, calcium and potassium are the largest component of trace elements, followed by iron, zinc and manganese. Meanwhile, the ratios between malignant and healthy tissue for these elements are higher in comparison with the other elements. Therefore, these six elements were selected to identify malignancy and reconstruct the images.

\subsection{Malignancy identification}

Using the above-mentioned trace elements, breast images were reconstructed. Figure 3 shows the reconstructed images of slice 3, after 20 iterations of the MLEM algorithm. In the images obtained with nitrogen, hydrogen and carbon as regular

1 National Nuclear Data Center. Brookhaven National Laboratory. 2.5 database, http://www.nndc.bnl.gov/nudat2/ elements and chlorine as a trace element with a low concentration ratio between malignant and healthy tissue, the pixel intensity is uniform through the breast volume, while the breast boundary can easily be distinguished. Among all trace elements, only potassium and sodium images can be used to distinguish the region of the lesion, which is qualitatively hotter than the other regions. Since the pixel intensity is directly related to the concentration of isotopes in a given organ, it confirms the higher accumulation of potassium and sodium in the malignant lesion, as expected from Table 1. However, the lesion boundary in the image obtained with sodium is not distinguished properly.

For quantitative determination of malignancy, the values of the contrast, CNR and mean pixel intensity, calculated in the same ROIs of the breast and tumor, are shown in Table 2. The mean pixel intensity in each ROI was compared using the $t$-test. Two-tailed $p$-values $\ll 0.01$ (99.9\% confidence level) were considered very highly significant. As indicated, the ratios of pixel intensity for sodium, potassium, iron and zinc are found to be proportional to their concentration ratio between malignant and healthy tissue (see Table 1). Sodium and zinc were previously reported in the study of Kapadia et al. (2010). The maximum pixel intensity ratio is 2.446 for the images of the potassium concentration. Although the malignant stage of the lesion is identifiable with these elements, only the image obtained by potassium has a contrast high enough (42\%) to differentiate the tumor from its surrounding region. This is the greatest contrast value, which was followed by manganese and sodium, with a tumor-to-healthy tissue contrast of about $13 \%$.

\subsection{Tumor location, volume and boundary identification}

Figures 4 and 5 show the $3 \mathrm{D}$ reconstructed images of the tumor obtained from the potassium and sodium 

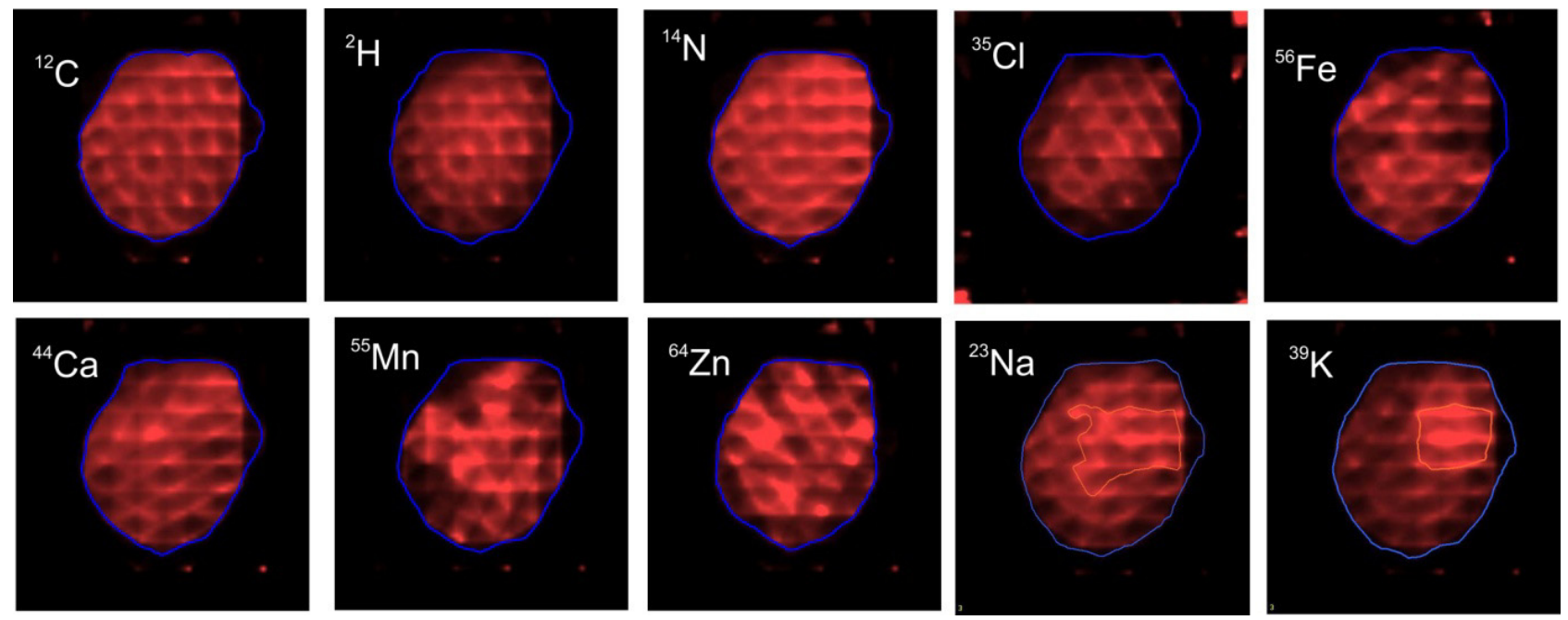

Figure 3. The reconstructed images of slice 3.

Table 2. The quantity of lesion contrast, CNR, mean pixel intensity and the ratio of that between malignant to normal tissues in the same ROI of breast and tumor shown in Figure 4 (blue circles). If the mean pixel intensity of breast and lesion are named $I_{\mathrm{b}}$ and $I_{1}$, respectively and the standard deviation of breast pixel intensity is $\sigma_{\mathrm{b}}$, the lesion contrast $(C)$ and $(C N R)$ are defined as $C=\frac{\left|I_{1}-I_{\mathrm{b}}\right|}{I_{1}+I_{\mathrm{b}}}$ and $C N R=\frac{C}{\sigma_{\mathrm{b}}}$, respectively. Two-tailed $P$-value was calculated from $t$-test for difference of means. Among all elements, the potassium created the greatest contrast. The mean pixel intensity of tumor versus that of breast for potassium is in agreement with the ratio of its concentration between malignant and healthy tissues.

\begin{tabular}{|c|c|c|c|c|c|c|}
\hline & \multirow[t]{2}{*}{ Contrast (\%) } & \multirow[t]{2}{*}{$C N R \times 10^{-7}$} & \multicolumn{2}{|c|}{ Mean pixel intensity \pm standard deviation } & \multirow[t]{2}{*}{ Mean pixel intensity ratio (tumor/breast) } & \multirow[t]{2}{*}{$P$-value } \\
\hline & & & ROI in breast & ROI in tumor & & \\
\hline Carbon & 5.6 & $1.59 \mathrm{E}-03$ & $2.08 \mathrm{E}-05 \pm 3.55 \mathrm{E}-6$ & $2.33 \mathrm{E}-05 \pm 3.77 \mathrm{E}-6$ & 1.119 & $\ll 0.01$ \\
\hline Hydrogen & 13.5 & 3.73E-03 & $1.22 \mathrm{E}-05 \pm 3.67 \mathrm{e}-6$ & $1.60 \mathrm{E}-05 \pm 3.88 \mathrm{E} 6$ & 1.313 & $\ll 0.01$ \\
\hline Nitrogen & 3 & 1.04E-02 & $1.66 \mathrm{E}-06 \pm 2.93 \mathrm{E}-7$ & $1.77 \mathrm{E}-06 \pm 2.98 \mathrm{E}-7$ & 1.062 & $\ll 0.01$ \\
\hline Chlorine & 13.4 & $5.60 \mathrm{E}-01$ & $6.47 \mathrm{E}-08 \pm 2.41 \mathrm{E}-8$ & $8.83 \mathrm{E}-08 \pm 2.81 \mathrm{E}-8$ & 1.309 & $\ll 0.01$ \\
\hline Potassium & 42 & $1.59 \mathrm{E}+00$ & $9.99 \mathrm{E}-08 \pm 2.65 \mathrm{E}-8$ & $2.44 \mathrm{E}-07 \pm 5.46 \mathrm{E}-8$ & 2.446 & $\ll 0.01$ \\
\hline Sodium & 9.3 & $3.47 \mathrm{E}-01$ & $1.03 \mathrm{E}-08 \pm 2.71 \mathrm{E}-8$ & $1.25 \mathrm{E}-08 \pm 3.24 \mathrm{E}-8$ & 1.206 & $\ll 0.01$ \\
\hline Iron & 1.8 & 2.19E-01 & $4.52 \mathrm{E}-08 \pm 8.19 \mathrm{E}-9$ & $4.68 \mathrm{E}-08 \pm 8.82 \mathrm{E}-9$ & 1.036 & $\ll 0.01$ \\
\hline Zinc & 3.8 & $2.27 \mathrm{E}+00$ & $5.43 \mathrm{E}-09 \pm 1.7 \mathrm{E}-9$ & $5.86 \mathrm{E}-09 \pm 1.69 \mathrm{E}-9$ & 1.080 & $\ll 0.01$ \\
\hline Manganese & 12.4 & $2.20 \mathrm{E}+00$ & $1.12 \mathrm{E}-08 \pm 5.66 \mathrm{E}-9$ & $1.43 \mathrm{E}-08 \pm 4.25 \mathrm{E}-9$ & 1.283 & $\ll 0.01$ \\
\hline Calcium & 5 & $1.66 \mathrm{E}+00$ & $1.12 \mathrm{E}-08 \pm 3.01 \mathrm{E}-09$ & $1.01 \mathrm{E}-08 \pm 4.58 \mathrm{E}-09$ & 1.110 & $\ll 0.01$ \\
\hline
\end{tabular}

concentrations. On the right side of these figures, the reference layouts of the voxel-based breast phantom are depicted and the boundaries of the reference tumor are superposed on the boundary obtained in both original reconstructed and binary images.

Qualitatively, it can be seen in Figure 4 (related to the potassium concentration) that the edges and location of the malignant region are in accordance with those in the reference images. Meanwhile, the tumor location is identifiable from the images of sodium. However, as seen in the 3D image in Figure 5 , the tumor boundary is larger than the reference boundary, and it extends to slice 6.

For quantitative examination, the volume of the breast and tumor, calculated before and after creating the binary images, is given in Table 3. From the image of the potassium concentration, the volume of the tumor was calculated with good accuracy, with a difference of less than $5 \%$ to the real tumor volume. This difference was improved to $3 \%$ when the tumor volume was calculated through the binary images. In addition, the volume obtained is larger than the real volume of the tumor. The effect of enhancing the visual representation after creating the binary image is clearly observed in the image of the sodium concentration. The huge difference between the calculated and real volume of the tumor in grayscale images was decreased by a factor of 5 in the binary images. Nonetheless, this difference remains in the order of $49 \%$.

The accuracy of detected boundaries in the reconstructed images was verified by examining the variations in pixel intensity of the tumor particularly. Pixels of the tumor edge were hence compared with pixels at the outer layouts adjacent to the edge. Figure 6 shows the normalized intensity on pixels which are along some of the cross-lines from the tumor in slice 3 of the image of the potassium concentration. As noted earlier, the pixel intensity of the reference image changes sharply at the edge of both the tumor and breast. Nevertheless, in reconstructed images, the variation of pixel intensity at the tumor edges is not sharp but has a steep gradient, as seen in Figure 6. 

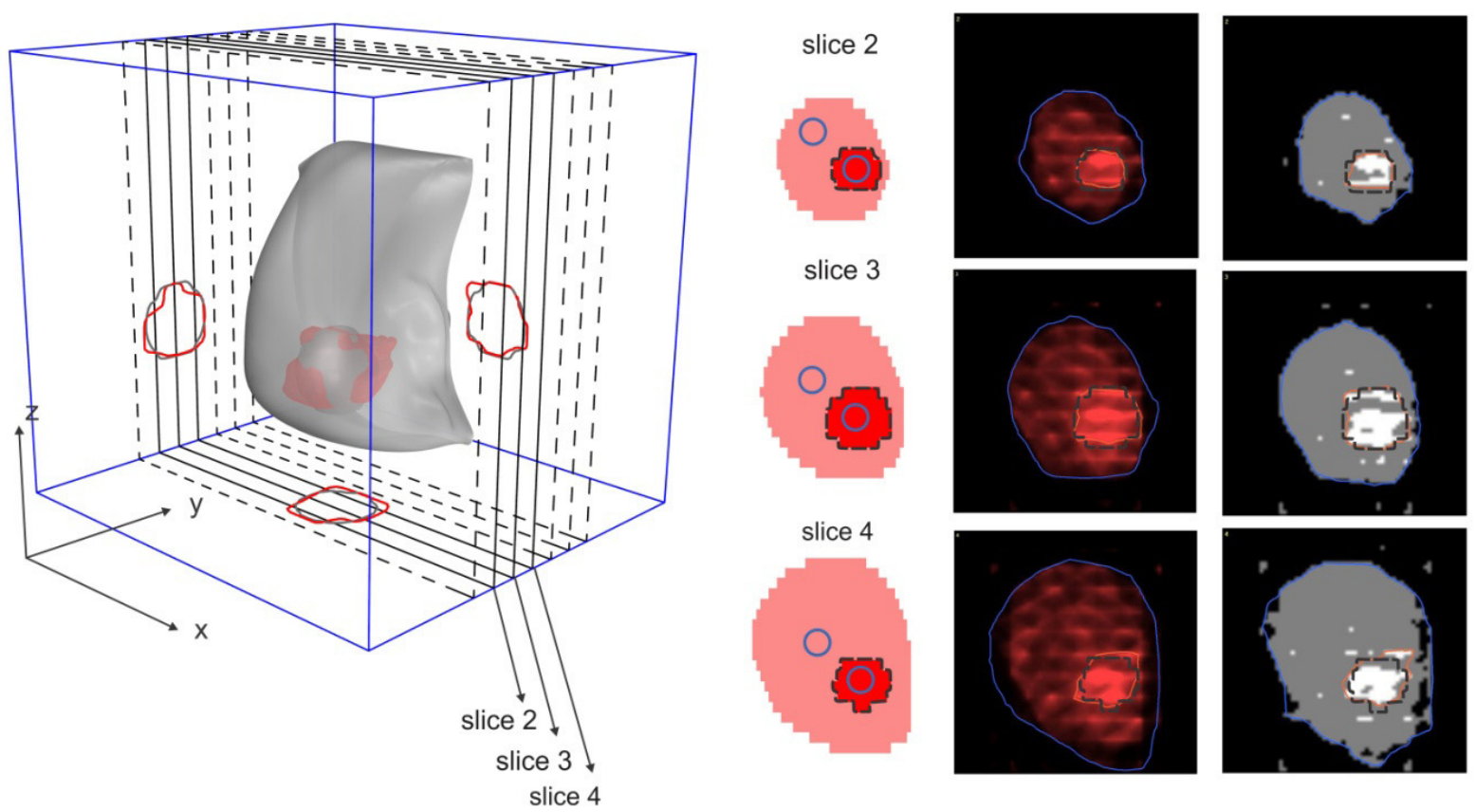

Figure 4. The reconstructed images obtained by ${ }^{39} \mathrm{~K}$ concentration. From left to right: three-dimensional image of tumor represented inside the breast sample, the irradiated layouts of the voxel-based breast phantom as reference images, the reconstructed images and stack of combinatorial binary images in the slices contained tumor (2, 3 and 4). In 3D images, the middle sections of the reconstructed volume and real tumor are shown in directions of $\mathrm{x}, \mathrm{y}$, and z-axes. The tumor boundary at the reference images was superposed on tumor at the reconstructed images. In reference images, the ROIs of breast and tumor determined for contrast and CNR calculation are shown with blue circles.
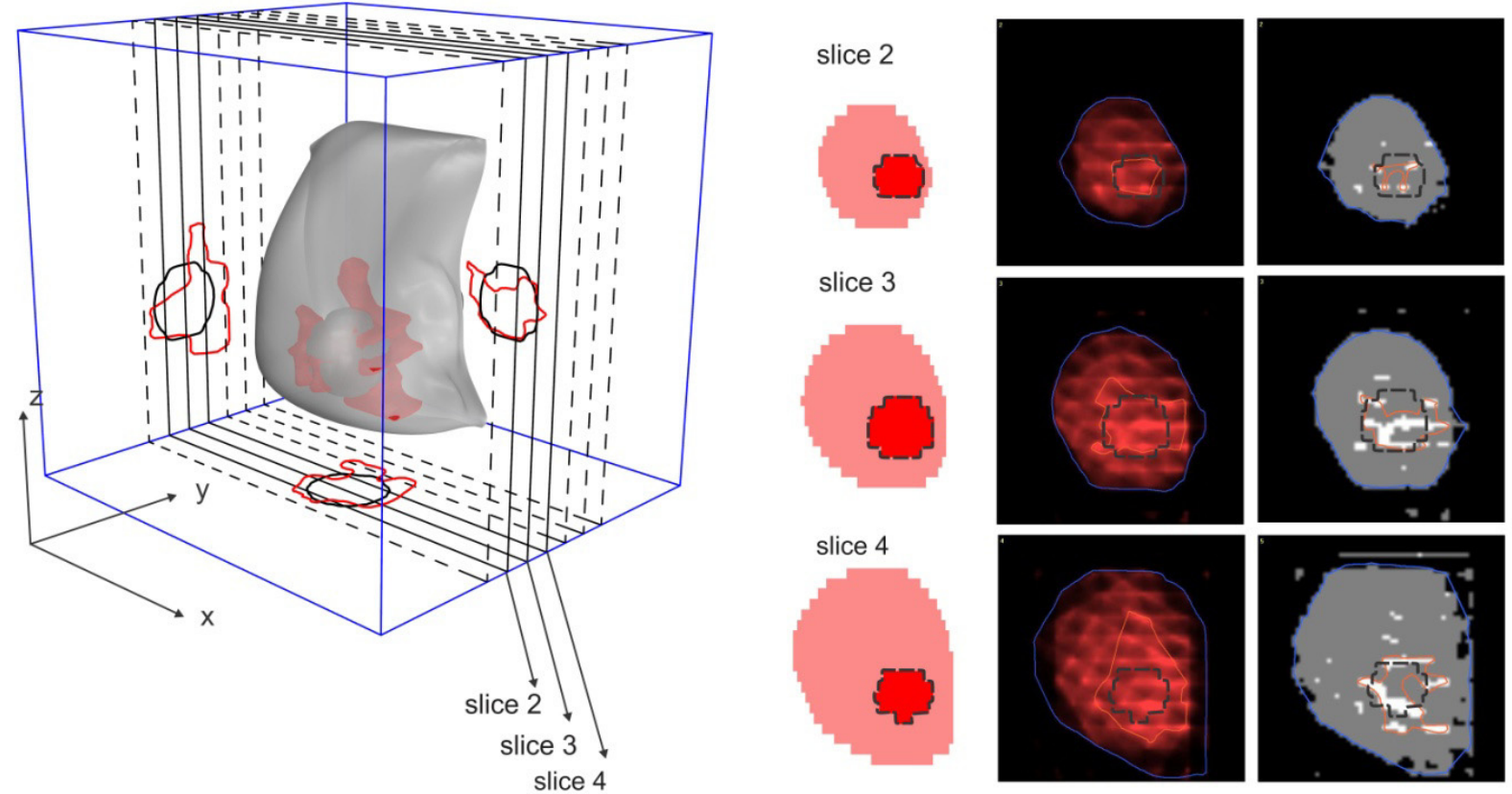

Figure 5. The reconstructed images obtained by ${ }^{23} \mathrm{Na}$ concentration. From left to right: three-dimensional image of tumor represented inside the breast sample, the irradiated layouts of the voxel-based breast phantom as reference images, the reconstructed images and stack of combinatorial binary images in the slices contained tumor (2, 3 and 4). In 3D images, the middle sections of the reconstructed volume and real tumor are shown in directions of $\mathrm{x}, \mathrm{y}$, and z-axes. The tumor boundary at the reference images was superposed on tumor at the reconstructed images. 
Table 3. The real volume of the simulated breast and its tumor compared with the volume calculated from 3D reconstruction before and after applying the binary mask. (Percent difference of the volumes was shown in bracket).

\begin{tabular}{cccccc}
\hline & \multirow{2}{*}{ Real volume } & \multicolumn{2}{c}{ Potassium } & \multicolumn{2}{c}{ Sodium } \\
\cline { 3 - 6 } & & Before & After & Before & After \\
\hline Breast & 264.14 & $284.81(7 \%)$ & $259.58(-1.73 \%)$ & $285.79(8.19 \%)$ & $260.11(-1.53 \%)$ \\
Tumor & 14.27 & $14.88(4.34 \%)$ & $14.65(2.67 \%)$ & $50.17(251.58 \%)$ & $21.26(48.98 \%)$ \\
\hline
\end{tabular}
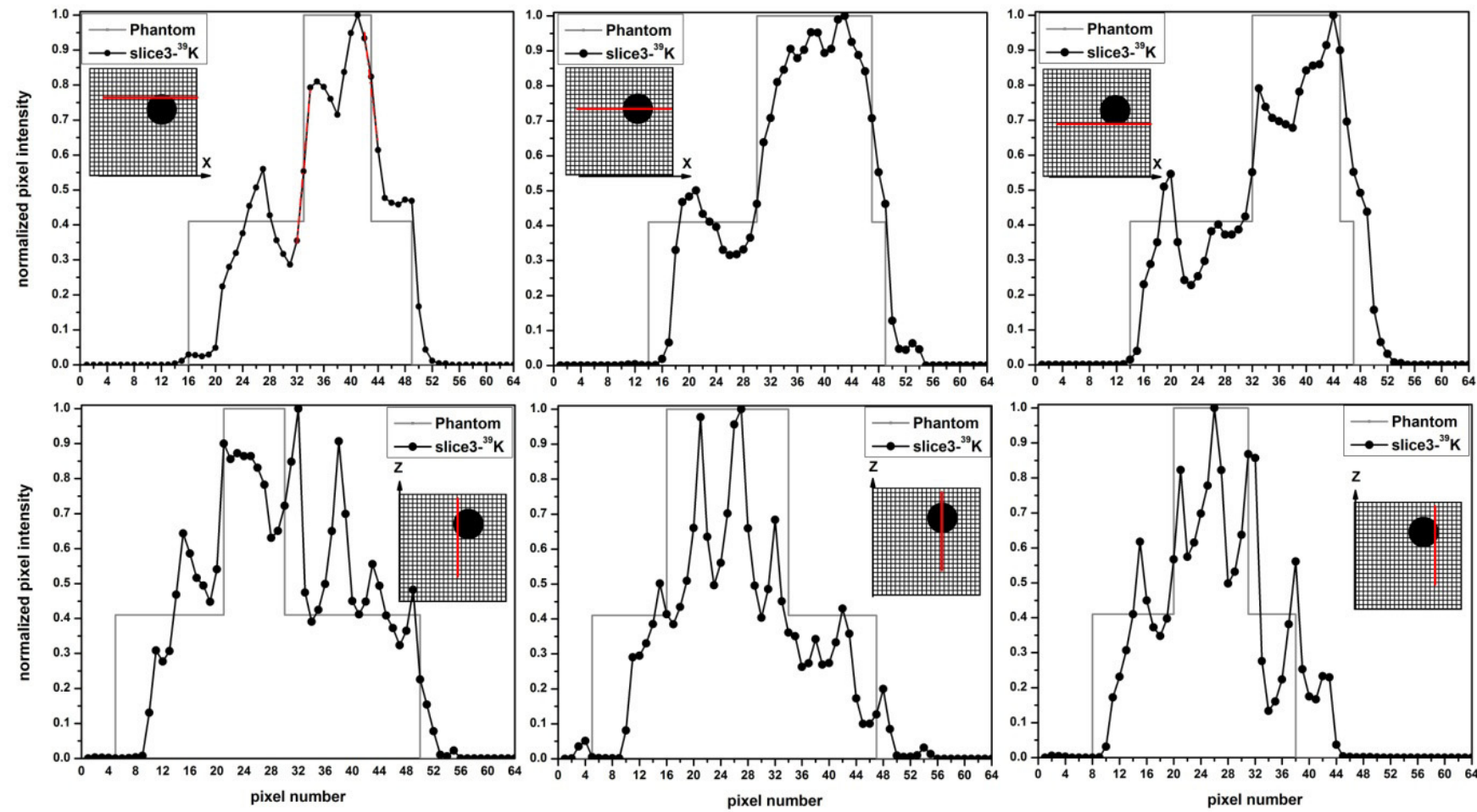

Figure 6. The normalized pixel intensities along some crossing lines from the tumor in $\mathrm{x}$ and $\mathrm{z}$ direction related to the slice 3 of image obtained by potassium concentration.

For more edge pixels, this steep gradient of variation is fitted to or goes beyond the true boundary of the tumor.

To generalize the outcome for all pixels at the tumor edge, the ratio of pixel intensity between the tumor edge and the outer layouts adjacent to the edge was calculated. The result of the cumulative probability calculation indicated that this ratio was higher than 1, for approximately $87 \%$ of edge pixels, and was higher than 1.5 for about $50 \%$ of edge pixels.

Accordingly, since most of the calculated ratios were higher than 1 , the edge of the tumor had a higher intensity than the outer part of the tumor. The proportion of calculated ratios which were close to 1 showed that the intensity of pixels adjacent to the tumor edge had the same order as the intensity of pixels at the edge itself. In these pixels, the boundary of the tumor went beyond the true reference edge of the tumor.

\subsection{Dose delivered to the breast}

In the present imaging system, the average absorbed dose in the target breast was $5.30 \times 10^{-10} \mathrm{mGy}$ per emitted neutron.
In order to calculate the total dose for a complete imaging of the breast, the number of neutrons required at each projection, the number of projections at each slice and the number of slices must be considered. Our assumption was in line with the studies of Kapadia et al. $(2004,2008)$ and Bender (2007) that 10 million incident neutrons at each projection were sufficient to provide a quantitative accuracy of $95 \%$. Therefore, the total absorbed dose of the target breast was calculated to be about 2.23 mGy $\left(5.30 \times 10^{-10} \frac{\mathrm{mGy}}{\text { neutron }} \times 10^{7} \frac{\text { neutrons }}{\text { beam projection }} \times 60 \frac{\text { beam projections }}{\text { slice }}\right.$ $\times 7$ slices). Considering the glandular tissue density, the average glandular dose (AGD) was evaluated to be about 1.1 times the average breast dose. Therefore, for a full breast scan, the average glandular dose would be about $2.44 \mathrm{mGy}$.

Considering the radiation and tissue-weighting factors (ICRP Publication 103, 2007), the breast and whole-body effective doses were calculated to be about 4.33 and $5.01 \frac{\left(10^{-10}\right) \mathrm{mSv}}{\text { neutron }}$, respectively. Therefore, following the same procedure of total absorbed dose calculation, the effective dose was calculated to be about $2.10 \mathrm{mSv}$ in the total scan. 
Table 4. The reference levels of AGD, the AGD in imaging method of digital mammography, 3D tomosynthesis and NSECT (this study) in mGy.

\begin{tabular}{|c|c|c|c|c|c|c|c|c|c|c|c|}
\hline \multirow{3}{*}{ ACR } & \multirow{3}{*}{$\begin{array}{l}\text { Limit levels } \\
\text { European protocol }\end{array}$} & \multicolumn{4}{|c|}{ Digital mammography } & \multicolumn{4}{|c|}{ 3D tomosynthesis } & \multicolumn{2}{|r|}{ This study } \\
\hline & & \multicolumn{2}{|c|}{ Standard breast phantom } & \multicolumn{2}{|c|}{ Real breast } & \multicolumn{2}{|c|}{ Standard breast phantom } & \multicolumn{2}{|c|}{ Real breast } & \multirow[t]{2}{*}{ AGD } & \multirow{2}{*}{ Total body Effective dose } \\
\hline & & $\mathrm{CC}$ & MLO & $\mathrm{CC}$ & MLO & $\mathrm{CC}$ & MLO & $\mathrm{CC}$ & MLO & & \\
\hline 3.00 & 2.50 & 1.57 & 1.66 & 1.82 & 1.94 & 2.19 & 2.29 & 2.53 & 2.63 & 2.44 & $2.10(\mathrm{mSv})^{*}$ \\
\hline
\end{tabular}

* Since the radiation-weighting factor for X-ray is equal to 1 , the value of absorbed dose is equal to equivalent dose in X-ray irradiation. Therefore, it can be compared with the effective dose in NSECT.

Table 4 compares the dose in the NSECT imaging method with the AGD in digital mammography and 3D tomosynthesis (Oglar et al., 2012) and the reference levels of the AGD (Hendrick et al., 1999; European Commission, 2006). As can be seen, the calculated doses in this study were lower than the recommended limit levels. In addition, our breast phantom similar to a real breast - had a lower glandularity proportion than the standard breast phantom. In conclusion, the AGD in our NSECT acquisition was about $4 \%$ lower than the AGD in $3 \mathrm{D}$ tomosynthesis. These small differences showed that the NSECT dose was comparable with mammography.

\section{Discussion}

\subsection{Identifying the malignancy of the tumor}

As observed in the gamma-ray spectrum of the breast, there are many peaks which prevent identifying the peaks of characteristic gamma rays of trace elements. Such a high background results from neutron capture interactions with some elements such as chlorine, and the inelastic scattering of nitrogen ${ }^{1}$. The moderating properties of hydrogen cause a neutron energy decrease following consecutive elastic collisions. By reducing the energy of neutrons, the probability of neutron capture interaction is increased.

The possibility of identifying the tumor properties at high background can be expounded considering Table 2 . The ratios of mean pixel intensity obtained for potassium, sodium, iron and zinc were in accordance with the concentration ratio of these elements between malignant and healthy breast tissue. These elements are the promising elements thanks to which the malignancy of a tissue can be predicted. Among these elements, the percent difference of concentration between malignant and healthy tissue is about $144 \%$ for potassium. This value is high enough to fit the sensitivity required for NSECT. In the present study, this value was confirmed by the ratio of mean pixel intensity between the lesion and breast for potassium (2.446).

For calcium, the expected contrast was about 20\% (Table 2) according to its concentration in malignant and healthy tissue (the malignant-to-healthy ratio is 1.52). However, the results indicated that using calcium, it was not possible to reconstruct the images of the lesion (the resulting contrast from imaging was $5 \%$, Table 2). This is due to the characteristic gamma rays of calcium, which are emitted with a probability of $100 \%$ with energy below $5 \mathrm{MeV}$. First, these gamma rays are restricted to some energy levels (about four energy levels). Second, they are missed in the background since their energies are very close to the energy of chlorine and nitrogen gamma rays. For instance, the peak of nitrogen at $3.948 \mathrm{MeV}$ has an overlap with calcium (Figure 2). Therefore, the uniform distribution of nitrogen in the breast resulted in erroneous estimation of the mean pixel intensity for calcium in tumor and breast regions.

\subsection{Identifying the location, size and true boundary of the tumor}

In order to detect the different regions in the reconstructed images, the concentration of the element used for imaging and its difference among these regions should be high in all regions and satisfy the sensitivity of NSECT for the following reason. Generally, based on the amount of materials presented in a LOC, the count of detectors was changed when the imaging system was transferred at different projections. For the elements with uniform distribution in the entire breast volume, detector counts had low fluctuations for different LOCs. Hence, the FOV was identically weighted by MLEM and the region with a malignant tumor could not be represented. Therefore, according to the previous discussion in Section 4.1, the image of the calcium concentration was not reconstructed due to the uniform distribution of nitrogen in the breast.

However, the difference in the count of gamma rays related to potassium in distinct LOCs was significant, and was satisfactory for the sensitivity of NSECT. Therefore, the region of the tumor would be brighter in the image obtained with this element. Sodium is the other element thanks to which it was possible to differentiate the tumor from the breast. However, the contrast with the sodium tracer was about 4.5 times less than that obtained with potassium. Figures 4 and 5 show an illuminated region that confirmed the lesion location in the image of the potassium concentration and showed the extended region in the image of the sodium concentration.

The accuracy of the reconstructed tumor and breast boundaries was shown using the calculation of volume in both sets of grayscale and binary images, and analyzing the ratio of pixel intensity at the tumor's edge to the outer layer of pixels adjacent to the edge. According to the results, as the images of the sodium concentration could not depict the true dimensions of the lesion, the volume of the tumor must be determined from the image providing the highest contrast. Following this approach, the lesion boundary was determined with good accuracy. Moreover, three results assured us that the cancerous region was completely detected, and therefore the need for a biopsy was eliminated. These results include the small difference between the calculated and real volume of the tumor, the greater volume obtained than the real one and the larger boundary of the tumor than the true boundary. In addition, according to the estimated dose in NSECT and the accuracy obtained 
in detecting the lesion, NSECT is a promising solution for younger women, for whom mammography is restricted.

\subsection{Limitations and future work}

This investigation showed that the malignancy, location and dimensions of an abnormal lesion could be determined with good accuracy (higher than 97\%) using NSECT. One of the most interesting issues in 3D breast imaging is the determination of the smallest detectable abnormal mass in the breast. By considering the parameters such as beam diameter, tumor diameter, depth of the tumor in the breast and size of the breast, the ability of NSECT to detect a mass with $1 \mathrm{~cm}$ diameter could be indicated (submitted for publication). Since the average size of breast cancer found on a mammogram is $1.1 \mathrm{~cm}$, it can be concluded that NSECT is appropriate for breast cancer screening.

In this investigation, the background of gamma spectra was not eliminated, in order to exhibit the ability of imaging by NSECT even in the presence of this high background. By employing background reduction techniques in practice, more trace elements can be used to quantify the properties of a suspected lesion (Kapadia et al., 2008). While the elements with a high concentration in both the lesion and breast are substantial markers for imaging, additional studies must be performed to increase the sensitivity of NSECT in detecting the elements at microgram levels of concentration and improving the contrast in the images of other elements.

As to the other limitations, certain concentrations of the body's elements should be determined due to the influence of some factors such as diet and environment on the amounts of that concentration. Therefore, a comprehensive database of the elemental concentration is required for all stages of breast cancer, which will render NSECT a practical achievable method for breast cancer diagnosis.

The calculated dose in this study showed NSECT to be a low-dose technique of potential great clinical use for breast screening. However, the value of the dose is affected by some parameters including the detectors' efficiency in recording the gamma rays, breast size and attenuation in neutron flux due to the interaction between neutrons and elements existing in the trunk. These parameters contribute to larger doses (about 1.43 times according to an initial calculation) as they directly impact the number of incident neutrons and image resolution.

\section{Conclusions}

This article investigates the feasibility of NSECT for breast cancer screening due to the fact that NSECT does not have the limitation of other screening techniques such as mammography. Using Monte Carlo simulations, a breast sample including a malignant tumor was examined in an imaging system which was designed to be close to reality. The malignancy, location and dimensions of the simulated lesion were determined with good accuracy (higher than 97\%). The malignancy of the lesion was verified using four trace elements: iron, zinc, and especially potassium and sodium. Three-dimensional imaging of the malignant lesion was achievable with potassium and sodium, and accurate boundary identification was obtained from the images of potassium thanks to the high contrast of the images. The calculated effective dose of the breast was less than the reference limit levels and was comparable with the dose of digital mammography and 3D tomosynthesis; therefore, the NSECT method fulfilled the purposes mentioned here.

Acknowledgements. We hereby acknowledge that parts of this computation were performed in the HPC center of Ferdowsi University of Mashhad. The authors also gratefully acknowledge Mr. Mehdi Zangian for his expert contribution and guidance, and MohammadJavad Mehrabifar, at Qaem MRI Center, Mashhad, for providing MR images required in this study.

\section{References}

Agasthya G., Shah J., Harrawood B., Kapadia A. (2011) Low dose, non-tomographic estimation of lesion position and trace element concentration in NSECT. In: The Nuclear Science Symposium and Medical Imaging Conference (NSS/MIC). IEEE, 2011.

Araghian N., Miri-Hakimabad H., Rafat-Motavalli L. (2015) Modeling the adult female phantom in the supine and prone postures and initial dose assessment in breast cancer diagnosis with Neutron Stimulated Emission Computed Tomography, Radioprotection 50 (2), 101-109.

Baptista M., Di Maria S., Oliveira N., Matela N., Janeiro L., Almeida P., Vaz P. (2014) Image quality and dose assessment in digital breast tomosynthesis: A Monte Carlo study, Radiat. Phys. Chem. 104, 158-162.

Bender J., Kapadia A., Sharma A., Tourassi G., Harrawood B., Floyd C. Jr (2007) Breast cancer detection using neutron stimulated emission computed tomography: Prominent elements and dose requirements, Med. Phys. 34 (10), 3866-3871.

Boyd N., Rommens J., Vogt K., Lee, Vivian, Hopper J., Yaffe M., Paterson A. (2005) Mammographic breast density as an intermediate phenotype for breast cancer, The Lancet Oncology 6 (10), 798-808.

Boyd N. et al. (2007) Mammographic density and the risk and detection of breast cancer, New Eng. J. Med. 356 (3), 227-236.

Chadwick M.B. et al. (2006) ENDF/B-VII.0: Next generation evaluated nuclear data library for nuclear science and technology, Nuclear data sheets 107 (12), 2931-3060.

Dempster A., Laird N., Rubin D. (1977) Maximum likelihood from incomplete data via the EM algorithm, J. R. Stat. Soc. B 39, 1-38.

European Commission (2006). European protocol for the quality control of the physical and technical aspects of mammography screening, European guidelines for quality assurance in breast cancer screening and diagnosis, 4th ed. EUREF (Luxembourg: European Commission).

Farquharson M.J., Geraki K., Falkenberg G., Leek R., Harris A. (2007) The localisation and micro-mapping of copper and other trace elements in breast tumours using a synchrotron micro-XRF system, Appl. Radiat. Isotopes 65 (2), 183-188.

Floyd C. Jr et al. (2006) Introduction to neutron stimulated emission computed tomography, Phys. Med. Biol. 51 (14), 3375-3390.

Harirchi I., Karbakhsh M., Kashefi A., Momtahen A. (2004) Breast cancer in Iran: results of a multi-center study, Asian Pacific Journal of Cancer Prevention 5 (1), 24-27. 
Hendrick R.E. et al. (1999) Mammography quality control manual: medical physicist's section, American College of Radiology, Reston.

ICRP Publication 89 (2002) Basic anatomical and physiological data for use in radiological protection: reference values, Ann. ICRP 32 (3), 1-277.

ICRP Publication 103 (2007) The 2007 recommendations of the international commission on radiological protection, Ann. ICRP 37 (2-4).

Kapadia A.J. (2011) In-vivo Diagnosis of Breast Cancer Using Gamma Stimulated Emission Computed Tomography. DTIC Document.

Kapadia A.J., Floyd C.E., Howell C.R., Harrawood B.P. (2004) Sampling Requirements for Neutron stimulated emission computed tomography. RSNA, Physics (Digital Imaging, PACS) session.

Kapadia A.J. et al. (2008) Neutron stimulated emission computed tomography for diagnosis of breast cancer, IEEE Trans. Nucl. Sci. 55 (1), 501-509.

Kapadia A.J., Shah J., Agasthya G. (2010) Quantitative elemental imaging with neutrons for breast cancer diagnosis: A GEANT4 study. In: The Nuclear Science Symposium Conference Record (NSS/MIC). IEEE, 2010.

Knoll G. (2010) Radiation detection and measurement. John Wiley \& Sons.

Leitch A. et al. (1997) American Cancer Society guidelines for the early detection of breast cancer: update 1997, CA: A cancer Journal for Clinicians 47 (3), 150-153.

Lovell M.A., Robertson J.D., Teesdale W.J., Campbell J.L., Markesbery W.R. (1998) Copper, iron and zinc in Alzheimer's disease senile plaques, J. Neurol. Sci. 158 (1), 47-52.
McCormack V., dos Santos S. (2006) Breast density and parenchymal patterns as markers of breast cancer risk: a meta-analysis, Cancer Epidemiology Biomarkers $\mathcal{E}$ Prevention 15 (6), 1159-1169.

$\mathrm{Ng}$ K.H., Bradley D.A., Looi L.M. (1997) Elevated trace element concentrations in malignant breast tissues, Br. J. Radiol. 70 (832), 375-382.

Olgar T., Kahn T., Gosch D. (2012) Average glandular dose in digital mammography and breast tomosynthesis, RoFo: Fortschritte auf dem Gebiete der Rontgenstrahlen und der Nuklearmedizin 184 (10), 911-918.

Pedraza V. et al. (2010) Gene expression signatures in breast cancer distinguish phenotype characteristics, histologic subtypes, and tumor invasiveness, Cancer 116 (2), 486-496.

Schwartz M. (1975) Role of trace elements in cancer, Cancer Research 35 (11 Part 2), 3481-3487.

Siddiqui M.K.J., Singh S., Mehrotra P.K., Singh K., Sarangi R. (2006) Comparison of some trace elements concentration in blood, tumor free breast and tumor tissues of women with benign and malignant breast lesions: an Indian study, Environ. Int. 32 (5), 630-637.

Van Schoor G., Moss S.M., Otten J.D.M., Donders R., Paap EDEN. (2011) Increasingly strong reduction in breast cancer mortality due to screening, Br. J. Cancer 104 (6), 910-914.

Viana R., Yoriyaz H. (2011) NSECT applied to the assessment of calcium deposition due to the presence of microcalcifications associated with breast cancer, Rev. Bras. Fis. Med. 5, 41-46.

Viana R., Agasthya G., Yoriyaz H., Kapadia A. (2013) 3D element imaging using NSECT for the detection of renal cancer: a simulation study in MCNP, Phys. Med. Biol. 58 (17), 5867.

Yaman M., Atici D., Bakirdere S., Akdeniz I. (2005) Comparison of trace metal concentrations in malign and benign human prostate, J. Med. Chem. 48 (2), 630-634.

Cite this article as: N. Araghian, H. Miri-Hakimabad, L. Rafat-Motavalli. 3D imaging of the elemental concentration associated with a malignant tumor in breast cancer using Neutron Stimulated Emission Computed Tomography: a Monte Carlo simulation study.

Radioprotection 51(2), 101-111 (2016). 